\title{
Comparative gene expression profiling of post-natal and post-pneumonectomy
} lung growth

\author{
J-C. Wolff*,\#, J. Wilhelm\#, L. Fink"\#, , W. Seeger*,\# and R. Voswinckel*,\#
}

ABSTRACT: Although increasing numbers of patients suffer from chronic destructive lung diseases, there are no effective therapeutic options apart from transplantation. Understanding the mechanisms of physiological and regenerative alveolar septation is prerequisite for the development of regenerative therapies for the lung. We compared lung gene expression in the phase of induction of post-natal and post-pneumonectomy alveolarisation to identify regulatory genes involved in both processes.

We performed genome-wide microarray screenings of newborn and pneumonectomised mouse lungs 1 and 3 days after birth or surgery. Selected candidates were validated by real-time PCR, Western blot and in situ hybridisation.

We found 58 genes to be regulated in both models with 40 candidates being changed likewise. Many of these genes participated in growth and differentiation processes. Additionally, immune system, structural molecules, respiratory chain, signal transduction and metabolism were involved. Some candidates were not yet linked to specific functions. The highest regulatory concordance was observed for various isoforms of (pro-)collagen molecules, elastin and the elastin-associated protein fibrillin1 being corporately upregulated.

Our findings do not definitively support a common regulating mechanism for induction of postnatal and adult alveolarisation, but some candidates in the intersection of both models are promising for further investigations.

\section{KEYWORDS: Alveolarisation, alveolar septation, arrays, gene expression, pneumonectomy}

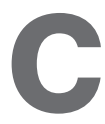

hronic destructive lung diseases, e.g. fibrosis, emphysema and chronic obstructive pulmonary disease, have become an increasing problem in today's pulmonary medicine. The severe alterations of the lung structure cause a continuously increasing affection of gas-exchange efficiency and an ongoing loss of quality of life. Although the number of patients is increasing, there are still very few therapeutic options for inhibiting or at least decelerating the tissue degradation and reorganisation processes. Often the only "cure" is lung transplantation, with all its inherent acute and chronic complications.

Therefore, strategies to overcome pulmonary tissue damage of patients by activation of endogenous regenerative programmes would be ideal. To our knowledge, this does usually not occur in humans, but the phenomenon of compensatory lung growth, which is a reinitiation of lung tissue formation in grown-up individuals, was already proven in many mammal models, e.g. mice, rats, dogs and rabbits [1-3]. In these animals, it was shown that unilateral pneumonectomy was not followed by a simple emphysema-like volumetric expansion, but that true alveolar growth and neo-alveolarisation reconstituted the amount of excised tissue. The tissue regeneration has been shown to be complete in rabbits, rats and mice. In the dog model, resection needs to exceed a threshold of $>50 \%$ before initiation of compensatory growth. All animals generate enough surface area to assure a normal life, even under physical stress [4]. One group demonstrated a regeneration of $\sim 50 \%$ of the excised alveoli in the residual lung 20 days after unilateral pneumonectomy of mice [5]. Reporting a model of lung tissue regression and regeneration, MASSARO et al. [6] showed the ability of mice to fully regrow alveolar surface area that was diminished during calorie restriction,

\section{AFFILIATIONS}

*Max-Planck-Institute for Heart and Lung Research, Bad Nauheim, \#University of Giessen Lung Center (UGLC)/Med. Clinic II, University Clinic of Giessen and Marburg $\mathrm{GmbH}$, Giessen, and

-Institute of Pathology and Cytology, UEGP, Wetzlar, Germany.

CORRESPONDENCE

J-C. Wolff

Max-Planck-Institute for Heart and Lung Research

Parkstrasse 1

D-61231 Bad Nauheim Germany

E-mail: Jens-Christian.Wolf@@

mpi-bn.mpg.de

Received:

Aug 092009

Accepted after revision:

Aug 132009

First published online:

Aug 282009 
again showing an inherent regenerative potential also under different circumstances.

These findings are promising enough to speculate about a reinitiation of lung growth in humans by stimulating or inhibiting the appropriate genes or pathways. To search for these candidates, we performed genome-wide RNA expression screens in two different mouse lung-growth models and analysed the data for corporately up- or downregulated genes that should have a high probability of being important elements for alveolar septation. With this intention, we investigated the gene expression in newborn mice undergoing physiological steps of alveolarisation and in adult 10-week-old animals undergoing compensatory lung growth after resection of the left lung. Post-natal lungs were compared with those of adult mice and post-pneumonectomy tissue with that of shamoperated animals. We chose a mouse model, as the possibility of performing consecutive studies with genetically engineered animals to analyse the physiological role of certain candidate genes is most intensively developed in mice.

\section{MATERIAL AND METHODS \\ Animals}

All experiments were performed according to the guidelines of good animal practice of the University of Giessen (Giessen, Germany) and were approved by the local authorities for animal experiments. We used adult (aged 10 weeks) and newborn (P1 or P3) C57BL/6N mice of both sexes, purchased from Charles River (Sulzfeld, Germany).

\section{Pneumonectomy}

For compensatory lung growth experiments, the left lung of adult mice was removed as described previously [7]. In brief, animals were anaesthetised using $3.5 \mathrm{~mL} \cdot \mathrm{kg}^{-1}$ of a $2: 1: 1$ mixture of $0.9 \% \mathrm{NaCl}$ solution and the anaesthetics ketamine (Ketavet ${ }^{\circledR}$; Pfizer, Karlsruhe, Germany) and xylazine (Rompun ${ }^{\circledR}$; Bayer, Leverkusen, Germany) as a single intraperitoneal injection. After disinfection and shaving, mice were intubated (21-gauge atraumatic cannula) and ventilated (MiniVent mouse ventilator; Hugo Sachs Elektronik, MarchHugstetten, Germany; $\left.150 \times 200 \mu \mathrm{L} \cdot \mathrm{min}^{-1}\right)$. Skin, muscle and fat tissue was incised in the left fifth intercostal space. After opening of the pleura, the left lung was lifted out of the thorax. Subsequently, all parts of the left hilus were ligated with $6 / 0$ silk and the lung was resected. After closing of the thorax and skin, mechanical ventilation was terminated at the onset of spontaneous breathing. All animals received a subcutaneous injection of $1 \mathrm{~mL}$ of $0.9 \% \mathrm{NaCl}$ as liquid substitution. During the days of recovery, mice were treated with antibiotics $(1 \mathrm{~mL}$ Baytril $_{\circledast}$ (Bayer)/500 mL drinking water) as well as subcutanous analgetics (buprenorphin, twice daily).

For sham-operated mice, the whole treatment was performed identically, but without ligation and resection of the lobe.

\section{Preparation of lungs}

Mice were sacrificed with an overdose of isoflurane (Baxter, Unterschleissheim, Germany). The trachea was incised and cannulated. A second cannula was inserted in the pulmonary artery. The apex of the left ventricle was incised, and the lung was flushed with $20 \mathrm{~mL}$ of $0.9 \% \mathrm{NaCl}$ solution at a constant pressure of $25 \mathrm{cmH}_{2} \mathrm{O}$ while being inflated with nitrogen gas via the trachea. Afterwards, the organ was removed and immediately frozen in liquid nitrogen. In newborn mice, flushing was performed with a needle via the right ventricle.

\section{RNA extraction}

To isolate total RNA from whole lungs, $10 \mu \mathrm{m}$ cryosections of up to $100 \mathrm{mg}$ of tissue were lysed in $1 \mathrm{~mL}$ peqGOLD TriFast ${ }^{\mathrm{TM}}$ solution (peqLab Biotechnologie $\mathrm{GmbH}$, Erlangen, Germany). After adding $200 \mu \mathrm{L}$ chloroform, 15-s shaking and 5-min incubation at room temperature, samples were centrifuged at $20,000 \times g\left(15 \mathrm{~min} / 4^{\circ} \mathrm{C}\right)$. The resulting upper phase was transferred into a new tube and mixed 1:1 with ethanol $(70 \%$ vol/vol). For RNA purification, we used the RNeasy Mini Kit (QIAGEN, Hilden, Germany) following the manufacturer's instructions.

RNA quantity was measured using the NanoDrop ND-1000 (Fisher Scientific, Schwerte, Germany); quality was assessed with the Agilent 2100 Bioanalyzer (Agilent, Boeblingen, Germany).

\section{Microarray analysis \\ Background}

We used microarrays spotted with about 44,000 different probes to which cDNA can bind in a highly sequence-specific manner. We always hybridised identical amounts of one control (adult, sham) and one experimental sample (post-natal, post-surgery) to the same chip, one being labelled with a red and the other one with a green fluorescent dye. In the case of identical expression (i.e. nonregulation), spots appeared in yellow; otherwise, they became more or less red or green, indicating a regulatory change in the direction of the dominating cDNA. By calculating expression ratios, we were able to generate context-dependent regulation profiles.

\section{Experimental design}

Profiles of differential gene expression were created by dual colour hybridisations comparing the expression profiles of 1) adult versus post-natal day $(\mathrm{P}) 1,2$ ) adult versus $\mathrm{P} 3,3$ ) sham versus post-pneumonectomy surgery day (S)1, and 4) sham versus S3. Each group consisted of 18 individuals. Total RNA from six individuals per group was pooled before labelling, resulting in three sample pairs for array hybridisation. Each pair was labelled and hybridised in a dye-swap manner (technical replications), resulting in 24 arrays in total.

\section{Labelling}

Cy-labelled cDNA was generated by reverse transcription of $50 \mu \mathrm{g}$ total RNA (pooled from six individuals) using the Superscript II reverse transcriptase kit (Invitrogen, Germany). Primer annealing was performed with $0.75 \mu \mathrm{g}$ oligo-dT primers in a volume of $18.5 \mu \mathrm{L}$ by heating the samples to $65^{\circ} \mathrm{C}$ for $10 \mathrm{~min}$ and subsequent chilling on ice. Reverse transcription was performed for $2 \mathrm{~h}$ at $39^{\circ} \mathrm{C}$ in $40 \mu \mathrm{L}$ Superscript first strand buffer containing $20 \mathrm{nmol}$ each of dATP, gGTP, dTTP, 8 nmol dCTP and 4 nmol Cy3- or Cy5labelled dCTP (Perkin Elmer, Waltham, MA, USA), respectively, $0.4 \mu \mathrm{mol}$ dithiothreitol and 300U Superscript II enzyme. The reaction was stopped by adding $10 \mu \mathrm{L} 1 \mathrm{M} \mathrm{NaOH}$ and heating to $65^{\circ} \mathrm{C}$ for $10 \mathrm{~min}$. The mixtures were neutralised by adding $10 \mu \mathrm{L} \mathrm{1M} \mathrm{HCl}$ and $200 \mu \mathrm{L}$ TE buffer (10mM Tris, $1 \mathrm{mM}$ EDTA, pH 8). Labelled cDNA was isolated using the PCR 
purification kit (QIAGEN) following the manufacturer's instructions.

\section{Hybridisation}

Labelled cDNA samples of the groups to be compared were mixed in in situ hybridisation buffer (Agilent Technologies, Waldbronn, Germany) and applied to G4122A microarrays (whole mouse genome $44 \mathrm{~K}$ arrays spotted with 60 mer oligonucleotide probes; Agilent). Hybridisation was carried out at $60^{\circ} \mathrm{C}$ overnight in Agilent rotating hybridisation chambers. Slides were washed with standard sodium citrate (SSC)/Triton-X-102 solutions according to the Agilent protocol.

\section{Scanning and image analysis}

Dried slides were scanned using the GenePix 4100A scanner (Molecular Devices, Ismaning, Germany) at a resolution of $10 \mu \mathrm{m} \cdot$ pixel $^{-1}$. Gains of the photomultipliers were adjusted to utilise the dynamic range and to obtain similar intensity histograms for both wavelengths. Image analysis was performed with GenePix Pro 5.0 software.

\section{Statistical analysis}

Extracted feature data were analysed in R [8] using the limma package [9] of BioConductor [10]. The spots were weighted for subsequent analyses according to spot intensity, homogeneity and saturation. Spot intensities were corrected for the local background using the method of EDWARDS [11] with an offset of 64 to stabilise the variance of low-intensity spots. The M/A data were LOESS-normalised [12] before averaging. Genes were ranked for differential expression using a moderated $\mathrm{t}$ statistic [13]. A power of $\sim 90 \%$ at a false-discovery rate of $10 \%$ was desired. Microarray data from a similar study (four arrays with technical dye-swap (= eight arrays in total), RNA pooled from six mice per sample) were available to perform a priori power tests. The required sample size (i.e. number of dye-swap pairs of arrays) was estimated based on these data using the method of FERKINSTAD et al. [14].

\section{Real-time PCR}

In order to validate the on-chip findings, real-time PCR was applied to six genes selected from the list of candidates found to be regulated in both experimental settings. For cDNA synthesis, reagents (Applied Biosystems Applera Deutschland $\mathrm{GmbH}$, Darmstadt, Germany) and incubation steps were applied as described previously [15]. Real-time PCR was performed using the 7900HT Fast Real-Time PCR system (Applied Biosystems, Foster City, CA, USA). Porphobilinogen deaminase was used as reference gene [16]. PCR reactions were set up using the Platinum SYBR Green qPCR SuperMixUDG (Invitrogen). Differential expression is given as $\Delta \Delta \mathrm{Ct}$ values [17]. Cycling conditions were as follows: $6 \mathrm{~min}$ at $95^{\circ} \mathrm{C}$ then $45 \times\left(5 \mathrm{~s}\right.$ at $96^{\circ} \mathrm{C}, 5 \mathrm{~s}$ at $59^{\circ} \mathrm{C}$ and $15 \mathrm{~s}$ at $\left.72^{\circ} \mathrm{C}\right)$. Products were checked by melting curve analysis and agarose gel electrophoresis. For primer data see table 1.

\section{Western blot analysis}

Proteins from lung homogenates were denatured in Laemmli buffer $\left(5 \mathrm{~min} / 95^{\circ} \mathrm{C}\right.$ ) and separated by sodium dodecyl sulfatepolyacrylamide gel electrophoresis using $10 \%$ polyacrylamide gels (stacking gels: $5 \%$ ) in a tank blot system (BioRad, Munich, Germany). Blotted membranes were blocked for $2 \mathrm{~h}$ with milk $/ 5 \%$ bovine serum albumin (BSA) in $1 \times$ phosphatebuffered saline (PBS). Primary antibodies (anti $(\alpha)$-Rras $2 / \alpha-c-$ fos: Santa Cruz Biotechnology Inc., Santa Cruz, CA, USA; and $\alpha$-Egr1: US Biological, Swampscott, MA, USA) were incubated overnight (in milk $/ 5 \%$ BSA, rotating, $4{ }^{\circ} \mathrm{C}$ ) and secondary antibodies (coupled to horseradish peroxidase) for $1 \mathrm{~h}$ (in milk $/ 5 \%$ BSA, room temperature, shaking). For visualisation of bands we used the Amersham ECL Plus Western Blotting Detection Reagents (GE Healthcare, Munich, Germany). $\beta$ actin (antibody from Sigma, St. Louis, MO, USA) served as loading control. Expression ratios were calculated with ImageJ (V1.41a).

\section{In situ hybridisation}

Generation of probes

Mouse lung cDNA fragments (400-700 basepairs) were subcloned into pGEM-Teasy vector (Promega, Madison, WI, USA). After sequence validation, $10 \mu \mathrm{g}$ of plasmids were cut using single restriction enzymes (Fermentas, St. Leon-Rot, Germany; $37^{\circ} \mathrm{C}$ /overnight; mostly Sall or SacII). Linearised products were cleaned up (PCR Purification Kit; QIAGEN) and transcribed into RNA probes using SP6 or T7 RNA polymerases (Promega; $37^{\circ} \mathrm{C} / 2 \mathrm{~h}$ ), respectively, introducing digoxigenin (DIG)-labelled UTPs (DIG RNA Labeling Mix; Roche) into the products. Finally, DNA was digested (RQ1 RNase-free DNase; Promega) and probe RNA purified (illustra ProbeQuant G-50 Micro Columns; GE Healthcare, Freiburg, Germany).

\section{Sampling}

Mouse lungs were flushed with PBS via the right ventricle and fixated with $4 \%$ paraformaldehyde (PFA; Carl Roth $\mathrm{GmbH}$, Karlsruhe, Germany; $25 \mathrm{cmH}_{2} \mathrm{O}$ ) via the trachea for $25 \mathrm{~min}$. Removed lungs were fixated in $4 \%$ PFA $\left(4^{\circ} \mathrm{C} /\right.$ overnight $)$ and dehydrated, followed by an overnight incubation in butanol, paraffination and embedding. Lungs were cut into $12-\mu \mathrm{m}$ slices and mounted onto SuperFrost Ultra Plus slides (Thermo Scientific, Schwerte, Germany).

\section{Hybridisation}

Samples were deparaffinised and rehydrated, followed by a proteinase K digestion. After washing, slices were fixated with PFA/0.1\% glutaraldehyde (Sigma-Aldrich, St. Louis, MO, USA) for $20 \mathrm{~min}$ and washed with PBS. Next, samples were pre-incubated in hybridisation mix $\left(2 \mathrm{~h}, 70{ }^{\circ} \mathrm{C} ; 50 \%\right.$ (vol/vol) formamide $+25 \%$ SSC $(20 \times, \mathrm{pH}=4.5)+20 \% \mathrm{H}_{2} \mathrm{O}+1 \%$ Tween 20 (Sigma-Aldrich) with $1 \%$ (weight/vol) blocking reagent, $5 \mathrm{mM}$ EDTA, $1 \mathrm{mM}$ Chaps, $20 \mu \mathrm{g} \cdot \mathrm{mL}^{-1}$ heparin and $1 \mathrm{mg} \cdot \mathrm{mL}^{-1}$ tRNA), followed by an overnight-incubation with preheated probes $\left(1 \mathrm{ng} \cdot \mu \mathrm{L}^{-1}\right.$, in hybridisation mix) at $70^{\circ} \mathrm{C}$. Afterwards, slides were washed $(2 \times \mathrm{SSC}, \mathrm{pH}=4.5)$ and incubated in $2 \times \mathrm{SSC} / 50 \%$ formamide $\left(2 \times 15 \mathrm{~min}, 65^{\circ} \mathrm{C}\right)$. Unspecifically bound probes were removed $(3 \times 10 \mathrm{~min}$ PBT $(\mathrm{PBS} / 0.1 \%$ (vol/vol) Tween 20)), followed by $1 \mathrm{~h}$ with blocking solution $\left(37^{\circ} \mathrm{C} ; 0.2 \%\right.$ blocking reagent (Roche) $+10 \%$ goat serum in PBT) and $2 \mathrm{~h}$ of an alkaline phosphatase-coupled anti-DIG antibody (1:1,000; Roche, in blocking solution). After washing, slides were incubated with NTM $(10 \mathrm{~min}, 100 \mathrm{mM} \mathrm{NaCl}+100 \mathrm{mM}$ Tris $\left.(\mathrm{pH}=9.5)+50 \mathrm{mM} \mathrm{MgCl}_{2}\right)$. Then, BM Purple substrate solution (Roche) was applied and incubated overnight. After 


\begin{tabular}{|c|c|c|c|c|c|c|}
\hline Gene & $\begin{array}{c}\text { GenBank accession } \\
\text { number }\end{array}$ & Forward primer & Exon & Reverse primer & Exon & $\begin{array}{l}\text { Product } \\
\text { length }\end{array}$ \\
\hline Egr1 & NM_007913 & gagcgaacaaccctatgagca & 1 & caaccgagtcgtttggctg & 2 & 109 \\
\hline Len2 & NM_008491 & tgcggtccagaaaaaaacaga & 2 & atccagtagcgacagccctg & 3 & 129 \\
\hline c-Fos & NM_010234 & gcctttcctactaccattcccc & 1 & aaagttggcactagagacggacag & 2 & 104 \\
\hline Rras2 & NM_025846 & gaggcatcggcaaagatcag & 4 & gttggttctggtgaaggaggg & 5 & 107 \\
\hline Fstl1 & NM_-008047 & atggcgactctcacctggac & 7 & caatgagggcgtcaacacag & 8 & 135 \\
\hline PBGD & NM_013551.2 & atgtccggtaacggcggc & 1 & ggtacaaggctttcagcatcgc & 3 & 139 \\
\hline
\end{tabular}

final washing with PBS, slides were mounted; target RNA molecules appeared in purple.

\section{RESULTS}

\section{Array analysis}

We found by far more significantly regulated genes (with $B$ values $\geqslant 0)$ when comparing newborn with adult mice $(1,669$ candidates for P1 and 2,032 for P3, respectively) than in the surgery model $(S 1=183$ and $S 3=161)$, probably due to the extreme difference in maturity in the former collective. Figure 1 gives an impression of the statistical data (volcano blots showing the correlation between $\log _{2}$-fold changes in mRNA expression and the probability of regulation and DE (differentially expressed genes) blots correlating inner-model changes at different time-points) and exemplarily depicts the degree of intersection of the top regulated genes of both timepoints in each model. We found 40 of the top 50 most significantly differentially expressed genes to be changed in both post-natal time-points, while there were only 10 intersection candidates in post-surgery lung growth (fig. 1). All genes found to be changed 1 and 3 days after birth or surgery, respectively, were uniformly up- or downregulated; none showed a switch.

\section{Candidate genes}

Figure 2 gives an overview of the functions of the top 50 up- or downregulated genes found in each group (see PubMed/ Entrez Gene (www.ncbi.nlm.nih.gov/sites/entrez) and GeneOntology (www.geneontology.org)). Categorisation was performed irrespective of promoting or inhibitory effects of the molecules studied here. The most striking finding was a unique dominating function or combination of functions in each condition. In newborn mice, we found predominantly growth/differentiation and translation genes as well as enzymes and their inhibitors to be upregulated (fig. 2a), while a large group of genes ascribed to the immune system and defence mechanisms was most intensively downregulated in newborn compared with adult lungs (fig. 2b). Similar to newborn mice, post-pneumonectomy lung growth was characterised by an involvement of growth processes and enzymes. Interestingly, we also found a non-negligible number of upregulated immune response genes in this category, although the sham-operated animals in the control group were presumed to "neutralise" this effect (fig. 2c). The genes being downregulated following surgical intervention were dominated by molecules found in mitochondria, mostly members of the respiratory chain (fig. $2 \mathrm{~d}$ ).

We found several collagen molecules unexceptionally being upregulated in both models, namely 13 genes in the post-natal and six in the post-surgery group (overlap: five candidates). This strongly underlines the need of connective tissue generation in the growing lung.

\section{Intersection of gene lists from newborn and pneumonectomy mice}

In total, we found 58 genes with significantly altered expression in both experimental settings (in at least one timepoint each, see table 2). Nineteen of these were mutually upand 21 downregulated. The remaining 18 candidate genes showed either a higher expression in newborn and a lower amount in pneumonectomised animals (six) or the other way round (12).

An examination of the function of these genes revealed many candidates being involved in growth and differentiation, followed by variable/unknown roles and immunomodulatory tasks (fig. 3).

\section{Real-time PCR}

To check the accuracy of our array data, we performed realtime PCR controls of six candidates from the genes appearing in the intersection list of both growth models (table 2, fig. 3). We evaluated three randomly chosen candidates with consistent post-pneumonectomy and post-natal regulation and three with differing directions (fig. 4) and analysed the timepoints found to be significantly changed in the array data.

In total, we were able to reproduce all regulatory tendencies found in the array experiments, mostly with good accordance in the regulation factor.

\section{Western blot analysis}

As differential regulations on RNA level (see table 2 and fig. 4) do not necessarily mean a change of the appropriate protein content, we checked the expression levels of three proteins, namely Rras2 (related RAS viral (r-ras) oncogene homologue 2), c-Fos (FBJ osteosarcoma oncogene) and Egr1 (early growth response 1), in the same experimental settings where their precursors were detected to be altered. For all the target 

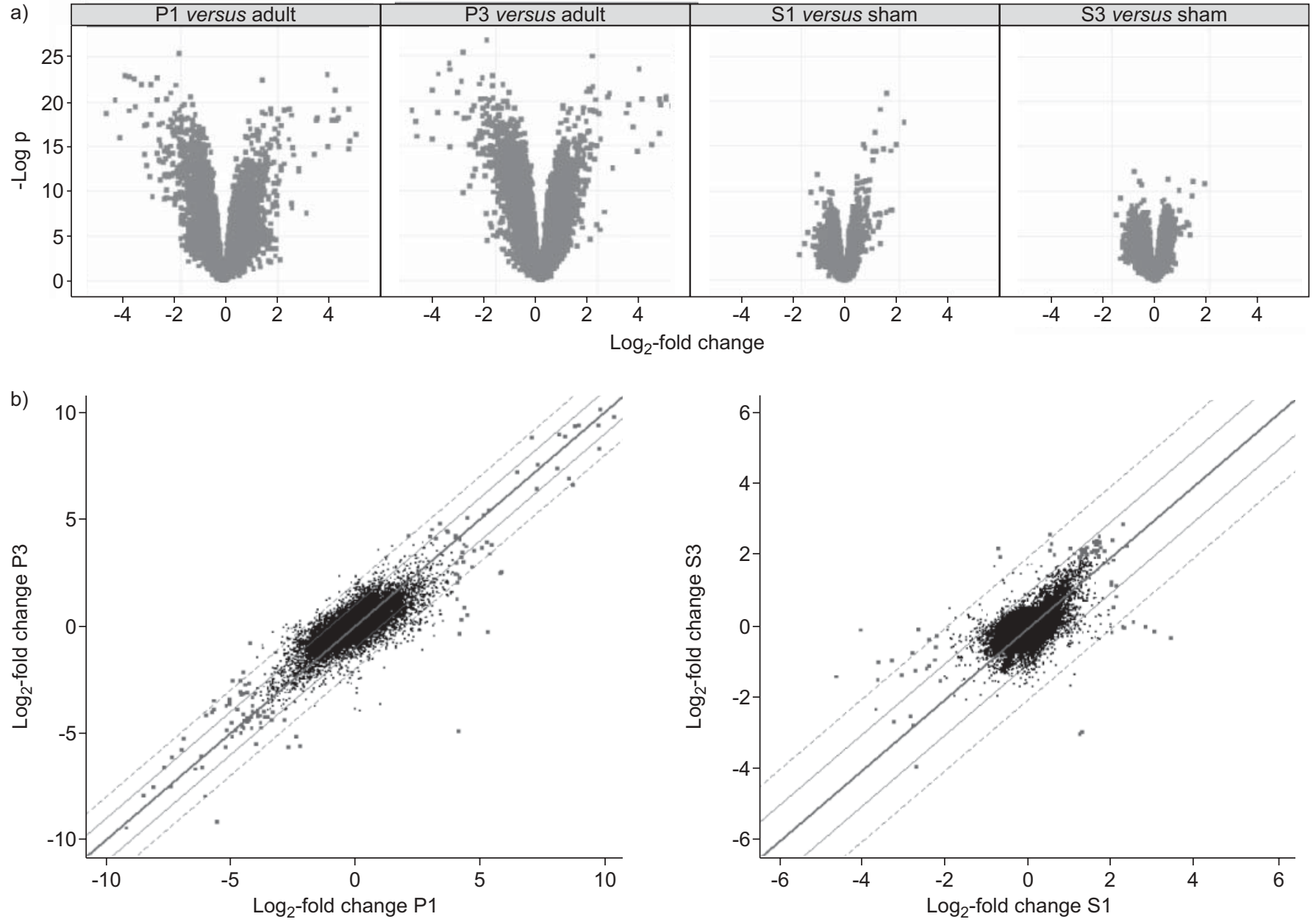

c)
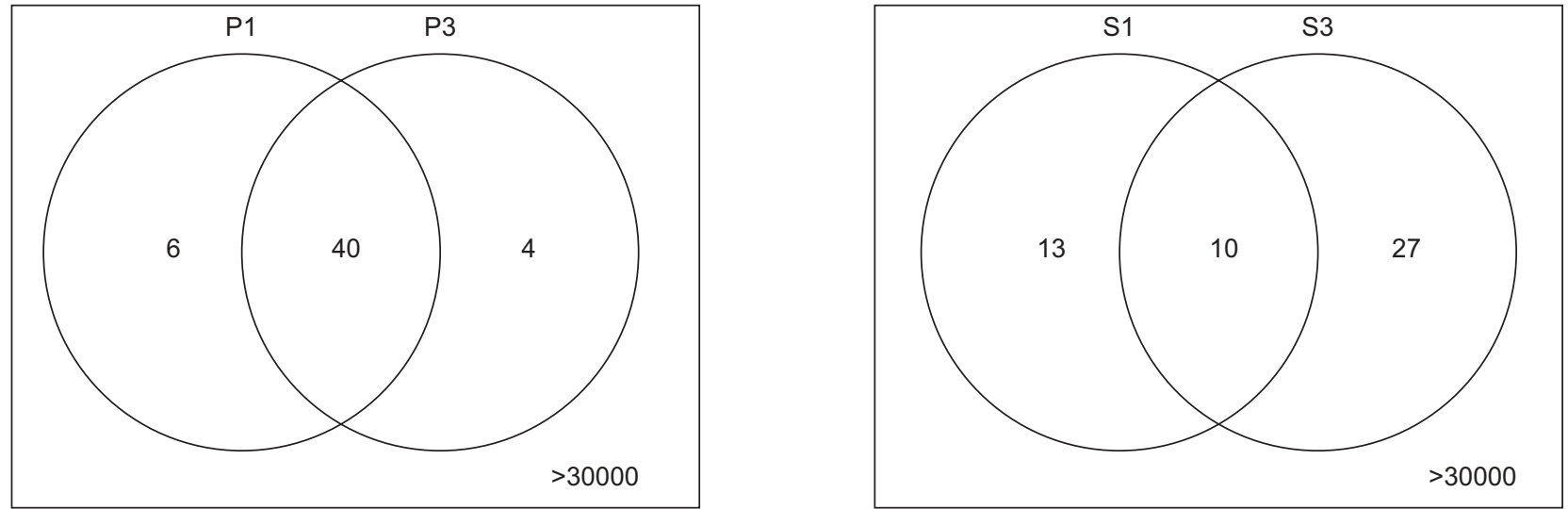

FIGURE 1. Statistical analysis. a) Volcano blots depicting regulatory change ( $\log _{2}$-fold) of all genes tested in all experimental settings ((P1 (post-natal day 1$) / P 3$ and $S 1$ (1 day after surgery)/S3) versus appropriate controls: adult and sham-operated mice, respectively) compared with the probability of regulation (-log(p)); negative log ${ }_{2}$-fold changes indicate downregulation and positive values indiacte upregulation. One spot represents the mean data of one probe on $\geqslant 6$ different arrays. b) DE blots (differentially expressed genes) comparing the regulation ( $\log _{2}$-fold changes) of all genes studied in P1/P3 and S1/S3 mice, respectively. Most post-natal candidates, if obviously changed at all (lying outside the central "cloud" around 0 values), showed very similar changes at both time-points (situated on or close to the bold line), while post-pneumonectomy genes were regulated more differently. Consequently, post-natal growth seems to be directed by the same genes for longer periods, while post-pneumonectomy growth is

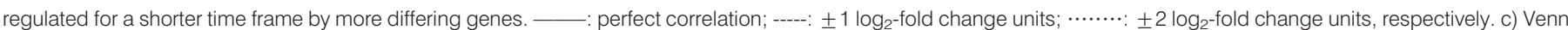
diagrams demonstrating the degree of overlap between the top 50 genes found to be regulated in P1 and P3, and S1 and S3. Far more candidates were found in both timepoints of post-natal lung growth, while, as in b), post-surgery genes seem to be regulated for shorter periods and appear only once. 

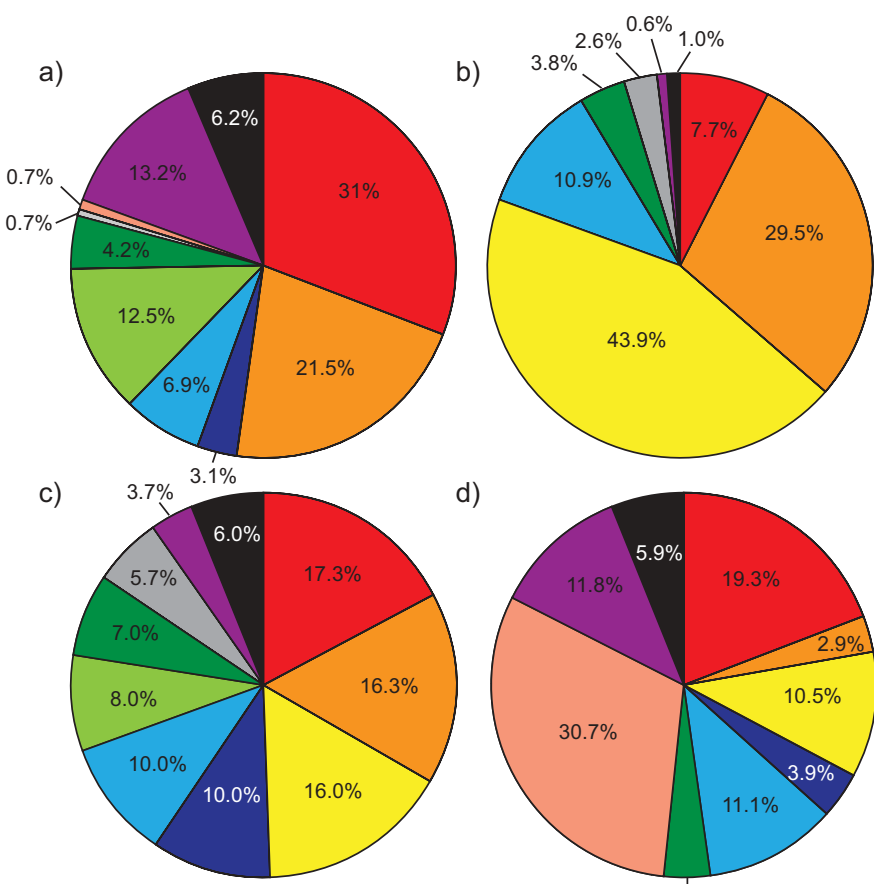

d)

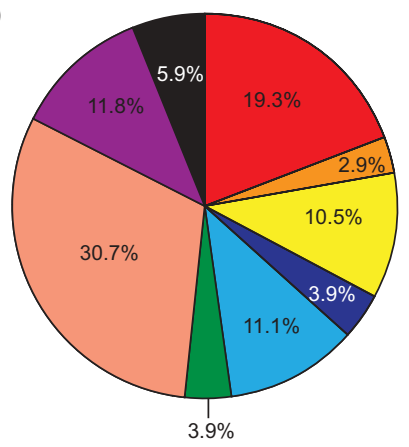

FIGURE 2. Functions of genes found to be significantly regulated post-natally (a) and b)) or after pneumonectomy (c) and d)). Each pie chart represents the distribution (percentages) of global molecular functions found to play a role 1 and/ or 3 day(s) after birth (newborn) or surgery according to PubMed/GeneOntology assignments. The top 50 most intensively up- (a) and c)) and downregulated genes (b) and d)), independent of whether changed after 1 or 3 days or both, were studied in each case. When more than one function was found, values were proportionally assigned. In every chart, at least one function is emphasised, e.g. growth molecules and enzymes being upregulated in newborn and members of the respiratory chain being less intensively expressed in surgery mice. @: growth/differentiation/ translation; 1 : enzymes and enzyme inhibitiors; 1 : immune system/defence; transport; $\mathbf{\square}$ : metabolism/ion binding; $\mathbf{\square}$ : structure molecules; $\mathbf{\square}$ : signal transduction and receptors; $\mathbf{\square}$ : reaction to external signals/detoxification; $\mathbf{1}$ : mitochondria/ respiratory chain; $\mathbf{\square}$ : others/not specified; $\mathbf{\square}$ : unknown.

molecules, we could validate the same tendency of regulation as found on microarrays (fig. $5 a$ and $b$ ).

A table with all array data is given in the online supplementary material.

\section{In situ hybridisations}

We exemplarily searched for the localisation of two of the PCR validation candidates, namely c-Fos and Lcn2 (lipocalin 2; acute phase reaction, epithelial morphogenesis) (fig. 5c-k). Both mRNAs were expressed in single septal cells in newborn mice and switched to a bronchial expression in adult animals. In the pneumonectomy model, we found an intensive upregulation of both candidates in single cells of the peripheral lung where the largest changes due to expansion can be expected.

\section{DISCUSSION}

\section{Experimental design}

We performed the gene expression screens in mice to further study candidate genes in genetically engineered animals in the future. For both post-natal and post-pneumonectomy lung growth we chose day 1 and day 3 for analysis. Our intention was to analyse gene expression in the induction phase of the responsible regulatory pathways, as this may be crucial for potential therapeutic interventions. P1 mice may be regarded as a measure of general lung growth; looking at table 2, we found 20 genes (among 58 in total) being differentially expressed at P3, but not at P1, while there were only eight candidates with opposite changes. This may be seen as a kind of specialisation (i.e. induction of new genes) directing to secondary septation as our target process. In contrast, we seem to have found an earlier induction phase in the compensatory growth model, as here even 37 (of 58) genes were exclusively regulated after 1 day.

\section{Previous investigations in the field}

Several other groups have also studied lung growth and regeneration in whole-genome approaches before, concentrating on specific aspects, medication effects or cell types, respectively.

MARIANI et al. [18] analysed a time-course from embryonic day 12 to adulthood focusing on extracellular matrix molecules and generating functional gene clusters. Here, one of the most interesting upregulated candidates in the post-natal phase was (tropo)elastin, which was also one of our most intensively altered molecules in post-natal as well as post-surgery animals (table 2). The group further analysed several collagens found to be more intensively expressed in either interstitial tissue or basement membranes. In both of our models, we could confirm the upregulation of interstitial type I and III collagens (table 2), while basement membrane collagen (IV) was only found to be slightly elevated 1 day after pneumonectomy (see online supplementary table).

FOSTER et al. [19] generated mRNA expression data from the tips of growing P6 septae isolated by laser-capture microscopy. Interestingly, only three of the 25 genes being most intensively upregulated were also significantly changed in our data (see online supplementary table), namely tenascin-XB and $C$ as well as drebrin 1, whereas tenascin-XB was downregulated in our lung homogenate. Also galectin-1, being the main focus of interest in this study, could not be confirmed by us. These differences in expression profiles may be due to the varying time-points of investigation (P1 and P3 versus P6), but we also have to take into account that using lung homogenate may hide certain findings, e.g. different cell types regulating genes in distinct directions due to varying needs at the same time.

Studying rat fibroblasts, BOUCHERAT et al. [20] searched for genes being up- or downregulated on P7 (phase of secondary septum formation) in relation to P2 and P21 [20]. Among the 141 candidates with significant changes, we found an overlap of 38 genes (27\%; compare with the online supplementary material). Of these, 35 were regulated only in the newborn model and 16 were altered in a different direction. Although the number of common genes was relatively high, we have to be careful in comparing these data as 1) a different species was used, 2) only a single cell type was studied, and 3) another (relative) time-point in septation has been chosen. This is also reflected by the 10 genes in the focus of interest of the study: Only Wnt5a, Fzd1 and Tnx were concordantly regulated in our 
TABLE 2 Selected genes found to be regulated in newborn and pneumonectomised mice

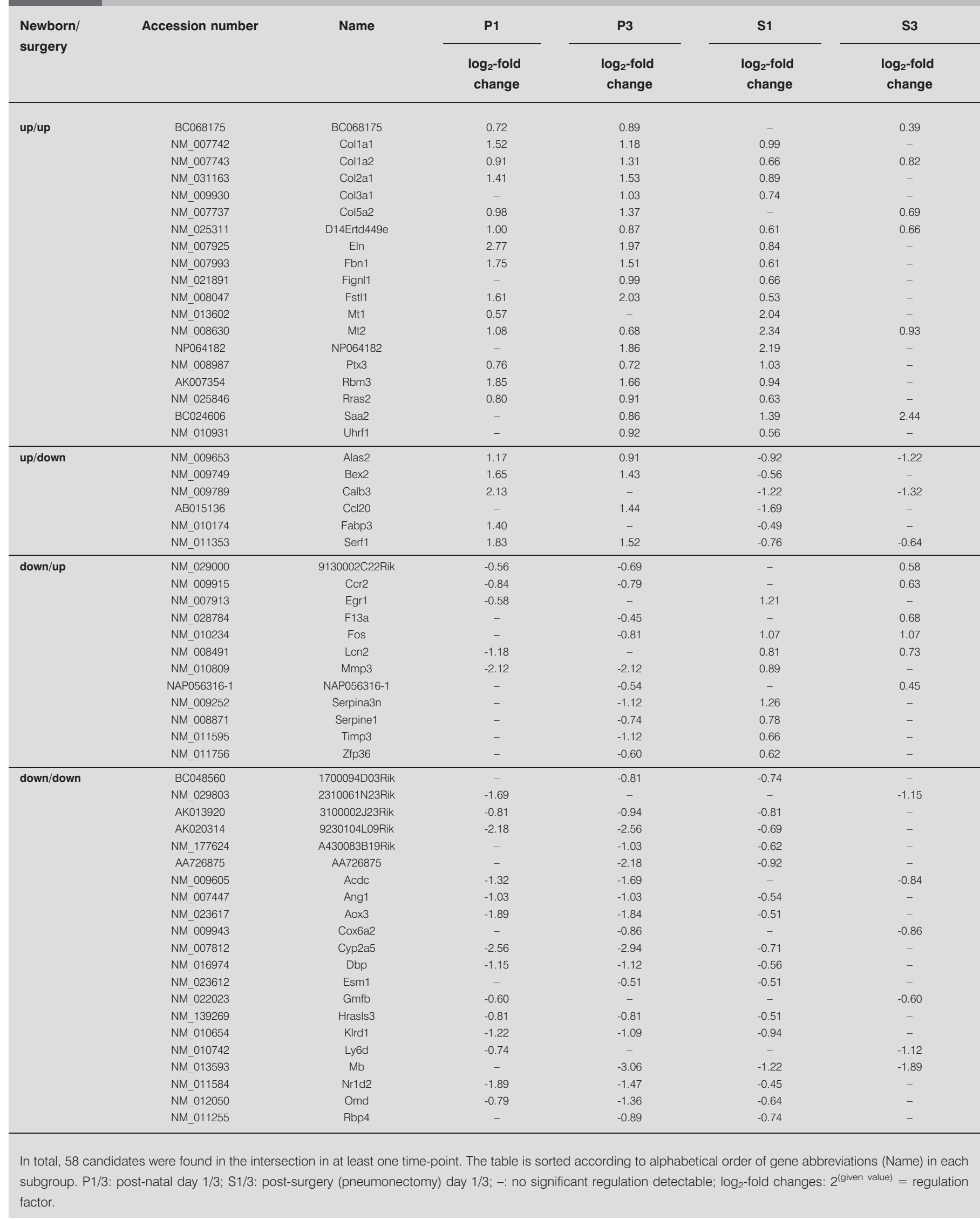




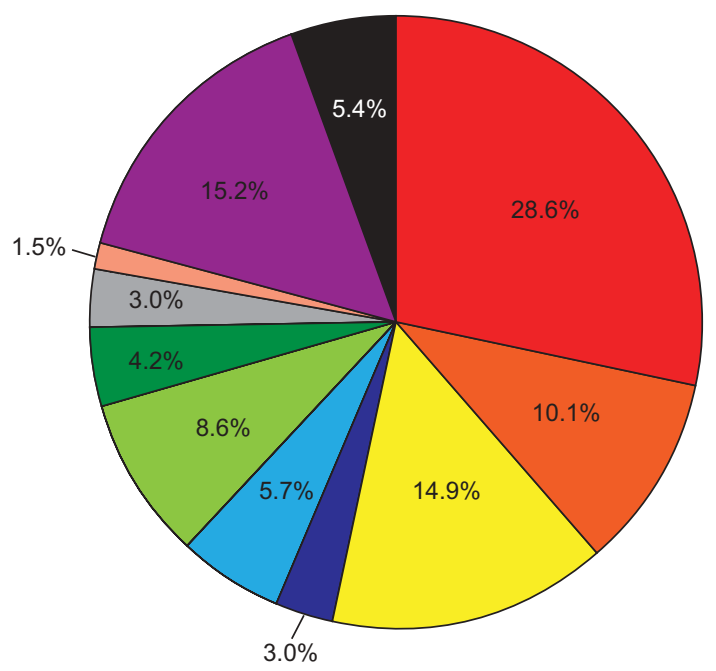

FIGURE 3. Functions of genes regulated post-natally and post-pneumonectomy. Distribution (percentages) of global molecular functions found to play a role in all 58 candidate genes (compare with table 2). Functions were assigned according to PubMed/GeneOntology, independent of the direction and the time-point of detected changes in array experiments. In case of more than one known function,

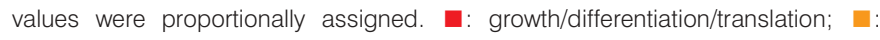
enzymes and enzyme inhibitiors; 1 : immune system/defence; $\mathbf{\square}$ : transport; $\mathbf{\square}$ : metabolism/ion binding; $\mathbf{\square}$ : structure molecules; $\mathbf{\square}$ : signal transduction and receptors; $\mathbf{a}:$ reaction to external signals/detoxification; 1 : mitochondria/ respiratory chain; $\mathbf{\square}:$ others/not specified; $\mathbf{\square}$ : unknown.

screening, while the remaining candidates, e.g. Ndp and Hox 1, 2 and 5, were not changed at all in homogenate.

MASSARO and Co-workers [21-23] performed several arraybased lung-growth studies, either on corticosteroid-triggered inhibition of alveolarisation in young mice or after calorierestriction and refeeding. In the former experiments, dexamethasone-treated animals were administered retinoic acid causing 46 drug-influenced genes to be "rectified" in their regulatory tendency [21]. We found only four of these candidates, namely Ly6a (lymphocyte antigen 6 complex, locus A), Chi313, Clca1 and Rnf2, to be significantly changed in normal post-natal (and none in post-surgery) mouse lungs, and only the latter two showed the same regulatory tendency. Especially Flk-1, being the main focus of MASSARO and coworkers [21], was not changed in our models. These findings support the assumption that corticosteroids seem to prevent lung growth by inhibiting pathways that are ongoing not only in secondary septation, but throughout the whole process.

In another series of experiments, the group focused on timerelated regulatory changes due to calorie restriction [22]. In our array lists, we found 10 out of $63(16 \%)$ apoptosis-related genes also to be changed in alveolar growth, with most of these candidates (e.g. Casp1 and 3, Birc2) being regulated in the opposite direction compared with early (4-h) lack-of-food effects. Regarding proteolysis-related genes, we even saw an overlap in 24 out of 78 genes $(31 \%)$, again with most of the candidates (e.g. Psmb8, Gzma and Gzmb) having contrary alterations. Having found so many common lung growth-related genes being changed in opposite directions due to opposite stimuli upgrades the validity of our data, as well as Massaro's data.

In a recent approach, the group intensively studied mice being re-fed after 2 weeks of calorie restriction with focus on angiogenesis-, extracellular matrix- and cell replication-related genes, as well as steering elements of guided cell motion [23] Altogether, we found a very high degree of overlap (between 30 and $50 \%$ ) throughout the groups, with special focus on angiogenesis and cell replication. The finding that several candidates showed a regulation in opposite directions may be caused by different stages of lung growth ongoing at the timepoints studied here (1- and 3-h refeeding compared with 1 and 3 days post-natally).

Regarding genes known to have an influence onto normal alveolarisation and/or compensatory lung growth (e.g. PdgfA, Hox, NeuroD, Fizz1 and eNOS), we only found Fibrillin1 to be significantly regulated. Reasons for this finding may, for example, be the overlapping profiles of different cell types in

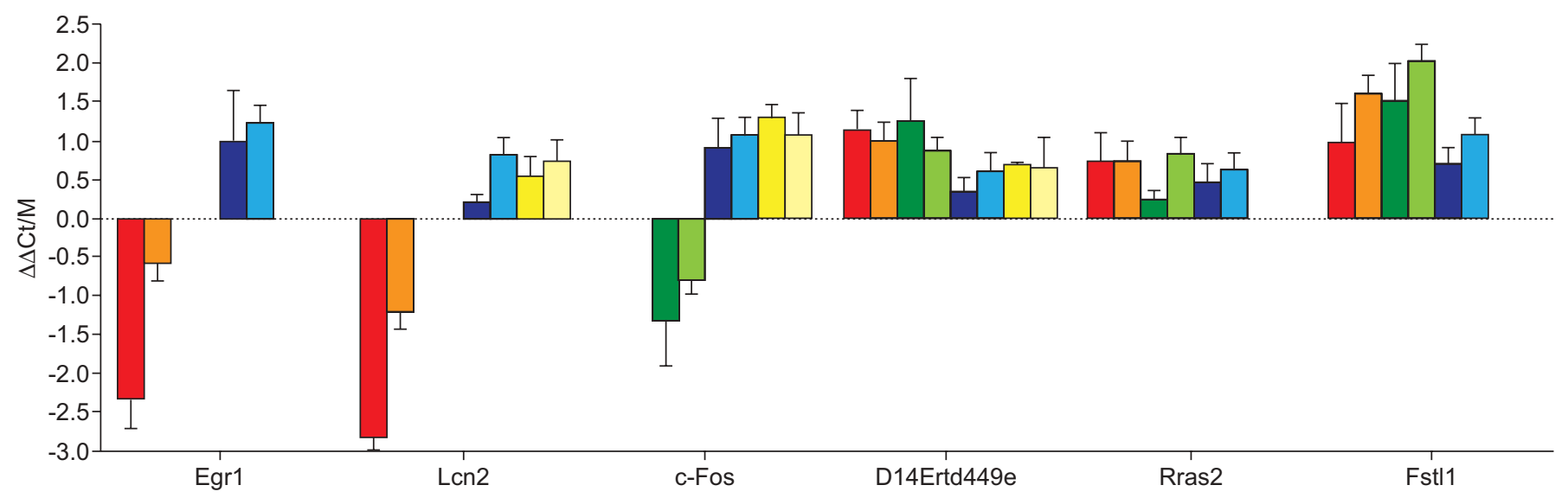

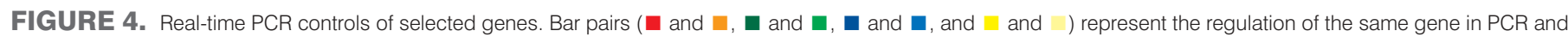

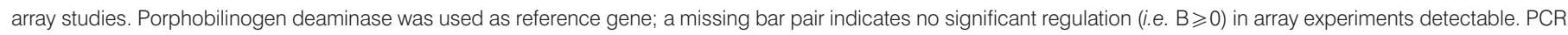

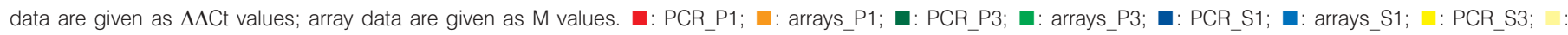
arrays_S3. $2^{\Delta \Delta C t}=2^{\mathrm{M}}$ : regulation factor; P1/3: post-natal days $1 / 3 ; \mathrm{S} 1 / 3: 1 / 3$ days after pneumonectomy. $\mathrm{n}=3-4$. Data are presented as mean \pm SEM. 
a)

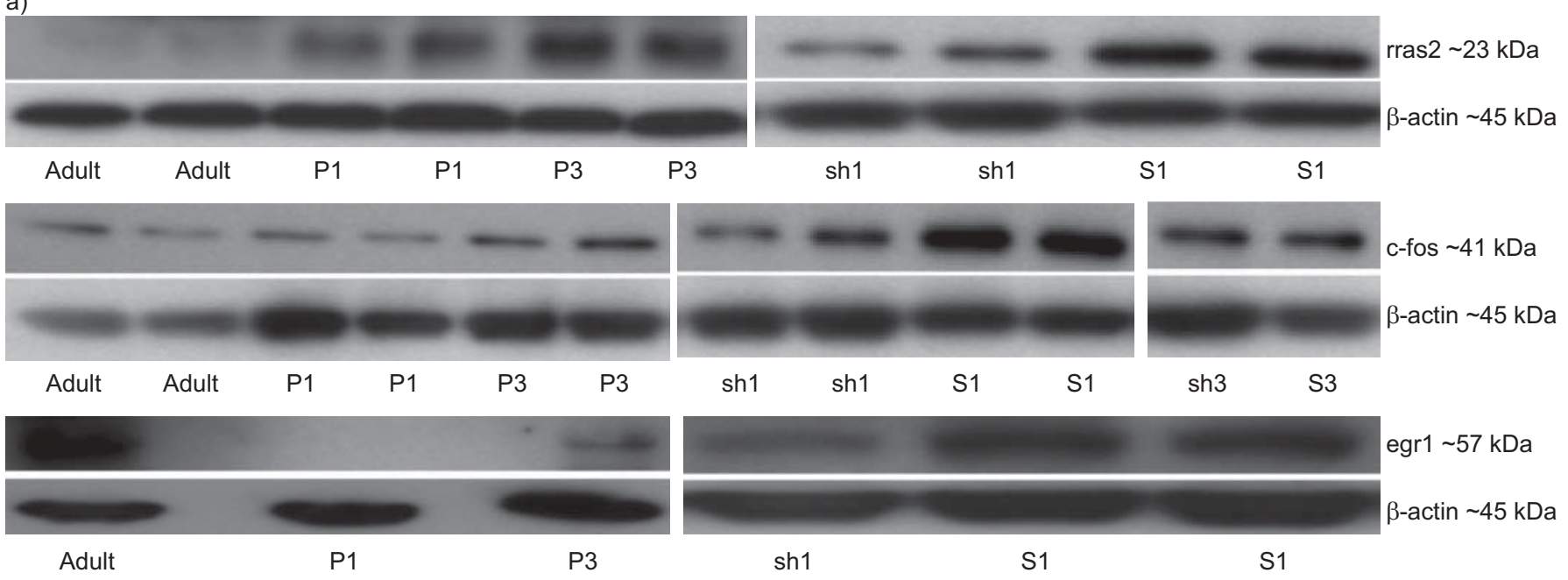

\begin{tabular}{|c|ccc|ccccccc|} 
b) & \multicolumn{3}{|c|}{ rras2 } & \multicolumn{3}{c|}{ c-fos } & \multicolumn{2}{c|}{ egr1 } \\
\cline { 2 - 11 } & Regulatory level & P1 & P3 & S1 & P1 & P3 & S1 & S3 & P1 & S1 \\
\hline mRNA & 1.74 & 1.88 & 1.55 & - & 0.57 & 2.10 & 2.10 & 0.67 & - & 2.32 \\
Proteins & 13.34 & 17.94 & 2.64 & $(0.52)$ & 0.74 & 1.47 & 1.44 & 0.03 & $(0.52)$ & 3.74 \\
\hline
\end{tabular}
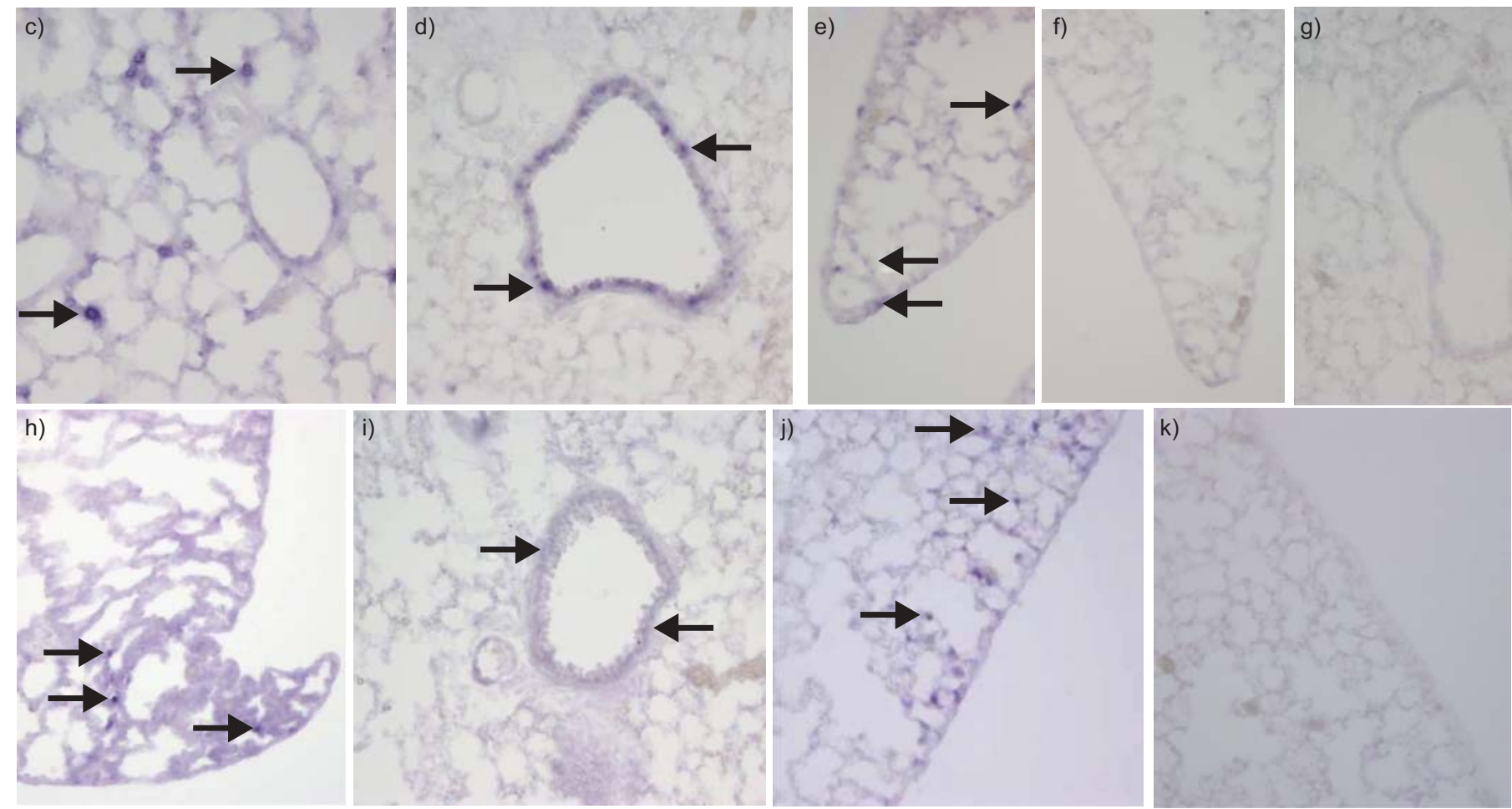

FIGURE 5. Protein expression and localisation. a) and b) Using Western blot, we checked the following three selected candidate genes (from PCR validation, fig. 4) for their protein expression: a) rras2, c-fos and egr1 blots, each with individual $\beta$-actin expression for normalisation. P: post-natal day(s); S: day(s) after surgery; Sh: day(s) after sham. b) Comparison of mRNA and protein expression for rras2, c-fos and egr1. Average regulation factors from array data and summarised Western blot bands (normalised by $\beta$-actin) are given. All regulatory tendencies could be validated; where significant array data are missing, blot data are given in parentheses. c)-k) Using in situ hybridisation, we found mRNA coding for c-fos to be intensively expressed in single septal cells of newborn mice (c, here: P3, similar in P1), but mainly in bronchial cells in adult animals (d). Post-pneunonectomy mice showed an upregulation of c-fos in single cells at the tip of a lobe (e, here: S1), which was missing in sham-operated animals (f, here: after 1 day). g) Control using c-fos sense instead of antisense probes ( $\mathrm{S} 1$ lung). For Icn2, we detected single, mainly peripheral septal cells to express specific mRNA (h, here: P1), while adult mice weakly expressed the molecule in bronchi (i). In compensatory growth, Icn2 was extremely upregulated in the peripheral lung (j, here: S1), but lacking in sham mice ( $\mathrm{k}$ here: after 1 day). Arrows exemplarily indicate peak expressions. All in situ pictures: magnification $200 \mathrm{x}$ 
our homogenate studies or different statistical and methodological approaches.

\section{Functional gene groups}

\section{Newborn mice}

The largest group of genes found to be upregulated was coding for molecules of growth and differentiation (31\%; fig. 2). All of these (e.g. Igf2 (insulin-like growth factor 2), Cdc2a (cell division cycle 2 homolog A), Tcf21 (transcription factor 21)), are known to have stimulating effects, fitting the necessities of growth promotion and organogenesis in this period of life. Additionally, there were some structural molecules being more intensively expressed than in adult animals (elastin, fibrillin), providing the stabilising and guiding scaffold for emerging tissue components. For the group of enzymes and their regulators, we found many molecules with inhibitory effects to be upregulated (e.g. StfA1 (Stefin A1), LOC268885), which mostly prevent matrix-degrading proteases from the destruction of essential, just-built extracellular matrix material, which is also plausible.

Conversely, there were several downregulated molecules with enzymatic activities, e.g. a procollagen endopeptidase enhancer (Pcolce2 [24]) or the proapoptotic allyldehydrogenase Aldh1a1; both of these findings also match the needs of growth and stabilisation. Another interesting point was to find 10 different cytochrome P450 oxidase (CYP) molecules to be downregulated. As these enzymes play a role in preventing damage through xenobiotics [25], one could speculate that in this early stage of life, interference by metabolism of xenobiotics would significantly damage the structure and that lower CYP levels prevent such damage. Regarding this point, the lack of Cyp2f2, which was downregulated 3.4-fold in P3 lungs, has been shown to have a protective role in naphthalene-induced Clara cell damage [26]. The downregulation of the microsomal epoxide hydrolase 1 (Ephx1), which also plays a role in detoxification, further supports this thesis. Conversely, it was recently shown that Clara cell-specific stabilisation of $\beta$-catenin leads to immature "progenitor"-like epithelial cells of the respiratory epithelium with significantly reduced expression of P450 cytochromes [27]. Therefore, a low CYP expression could also reflect the immature state of the epithelial cells during rapid cell expansion. Having found Xdh (xanthine dehydrogenase) and Ly6a, which both have a pro-differentiative influence, to be downregulated, additionally confirms the immature state. The most striking finding in the downregulated group were numerous genes involved in immune response mechanisms. The subgroups covered many areas of defence, e.g. antigen presentation via major histocompatibility complex molecules, leukocyte surface receptors and parts of the complement system. We speculate that the immune system is still in an immature state and comparably few inflammatory cells reside in the lung in this developmental period, which may be tolerable as long as the babies receive antibodies via milk. This could, as with the CYPs, also be happening "on purpose" to develop a certain degree of tolerance against own tissue components.

\section{Surgery model}

Among the upregulated genes, we found a relatively homogenous distribution of most global molecular functions. Again, the largest group of genes included growth and differentiation molecules, especially transcription factors or mediators of proproliferative pathways (e.g. Egr1, Fos). In addition, six different collagens were more intensively expressed (five of them in parallel with the newborn model; compare table 2), underlining the need of connective tissue generation in compensatory lung growth. Interestingly, we found some immunomodulatory genes to be induced, although sham operations were performed on control animals. Some of these may indeed be upregulated by potentially more severe wounding than in the control animals, but also genes with downregulating and/or secondary (side-)effects were detected, which may be emphasised in our model, e.g. Z49206 (= Hsf1, heat shock factor 1; inhibits tumour necrosis factor- $\alpha$ production and is a transcription factor) [28] or Lcn2 [29, 30]. Conversely, several stimulators of the immune system were also found to be downregulated, at least in part diminishing the effect of some of the former proinflammatory genes.

In the group of downregulated genes, we again found several growth and differentiation molecules, often with inhibiting functions (e.g. Fabp4 (= fatty acid binding protein 4; inhibits transcription) and Srp9 (= signal recognition particle 9; inhibitor of transcriptional elongation) [31]). A very interesting finding was to reveal several members of the respiratory chain/the mitochondrial metabolism being downregulated after pneumonectomy. At a first glance, this finding is surprising, as we can assume a high degree of energy demand during lung growth and the need to compensate about onethird of respiratory surface. One might think about a relative effect, as the mitochondria may not divide as quickly as their "mother" cells, but all of the 17 genes we found were encoded by the cellular genome and none by the mitochondria themselves. The downregulation may be explained by the autophagy phenomenon: For the heart, it is known that in periods of extreme demand, cells are able to "burn up" their mitochondria to quickly gain large amounts of energy and amino acids [32, 33]. Something similar may also be assumed in the onset of compensatory lung growth.

\section{Genes regulated in post-natal and post-pneumonectomy lung growth}

In total, we found 40 genes being concordantly up- or downregulated in both experimental groups and 18 candidates with opposite directions of change (table 2). Among the former genes, we can assume to have found some important regulators or operators of alveolarisation as the "accidental" finding of uniform expressional changes without any functional mutuality in the overlay of two independent models can be regarded as unlikely. The discordant genes have to be discussed in a more differentiated way: some, if not most of them may simply not play an important role in lung growth. Few candidates could mirror different time-points in a long regulatory pathway where opposing or completely distinct influences are needed, e.g. transcription factors like Egr1: this molecule can induce proliferation, differentiation, angiogenesis, induction and inhibition of apoptosis etc. [34-36]. All of these features are necessary for lung growth, but have to appear in the correct order.

Among the candidates in the intersection list, the by far largest functional group was "growth and differentiation": 10 out of 
$19(53 \%)$ corporately up- and nine out of 21 (43\%) downregulated genes were, at least in part, involved in these processes, e.g. Rbm3 (= RNA binding motif protein 3), which is necessary for mitosis progression [37]. Another example of upregulated genes is Fstl1 (follistatin-like 1, TSC36). The protein has an anti-apoptotic, heart-protecting effect and is thought to play a role in organogenesis. A generalised expression, becoming more and more localised, has already been described for embryonic mouse lung and kidney development [38]. With Uhrf1 (= ubiquitin-like, containing PHD and RING finger domains, 1 ; Np95) we found a molecule responsible for S-phase entry and genome stability to be elevated in both models [39]. Rras2 (TC21) is known to stimulate migration; in some cases, it can even induce tumours [40]. The detected upregulation in both models points to the need of cell migration for proper lung growth.

In addition to these growth-promoting factors, there were several structural molecules, which are essential for a proper lung architecture, concertedly also upregulated, e.g. five different collagens, elastin and fibrillin1.

Among the downregulated genes, we revealed several candidates responsible for maintenance procedures of differentiated cells, but also growth inhibiting molecules, e.g. Pla2g16 ( $=$ phospholipase A2, group XVI $=$ Hrasls3 $=\mathrm{H}-$ rev107), which is a well-known cell cycle inhibitor [41]. Again, these findings can be justified by the needs of proliferation and differentiation when lung growth is conducted.

Interestingly, the degree of overlap between both models was relatively small, although we found $>150$ (pneumonectomy) and $>1,500$ (post-natally) genes to be significantly regulated in each setting, respectively. There are two different explanations for this outcome: On the one hand, the whole alveolarisation procedure may be depending on very few initiating and/or executing molecules. In this case, we may have found at least most of the key genes. On the other hand, both models could be relatively independent, showing similar features regulated by different pathways. Then the idea of lung tissue regrowth in humans would have to be realised with genes from one or the other model, preferably with pneumonectomy candidates, as this issue is closer to what can be expected to happen in adults.

Our findings narrowed down the number of genes that can be regarded as potential candidates for septation regulation, but functional studies on these genes have to reveal their actual role and answer the question whether there is indeed a common background to both models.

In a first step, we could localise the expression of c-Fos- and Lcn2-specific mRNA in post-natal and post-surgery lungs (fig. 5). Both molecules showed a switch of expression being expressed in single septal cells after birth, but in the bronchi of adult mice. Regarding the surgery model, we found the specific mRNA to additionally appear in the periphery of pneumonectomised, but not of sham-operated animals. This may be explained by the need of the outer part of the lung to more intensively grow than internal regions and fits the clear upregulation of both genes we detected in array experiments (table 2).

\section{SUMMARY}

Studying two lung growth models, we used microarraybased screens to find genes with significant expressional changes being potentially involved in post-natal and/or post-pneumonectomy alveolarisation. While there were high numbers of candidates found especially in newborn mice, we only saw a small degree of model intersection, either due to the importance of few key regulators or because of a wide independence of the processes.

\section{STATEMENT OF INTEREST}

None declared.

\section{ACKNOWLEDGEMENTS}

The authors would like to thank D. Fuchs for skilful technical assistance.

\section{REFERENCES}

1 Schittny JC, Mund SI, Stampanoni M. Evidence and structural mechanism for late lung alveolarization. Am J Physiol Lung Cell Mol Physiol 2008; 294: L246-L254.

2 Foster DJ, Yan X, Bellotto DJ, et al. Expression of epidermal growth factor and surfactant proteins during postnatal and compensatory lung growth. Am J Physiol Lung Cell Mol Physiol 2002; 283: L981-L990.

3 Cagle PT, Langston C, Thurlbeck WM. The effect of age on postpneumonectomy growth in rabbits. Pediatr Pulmonol 1988; 5: 92-95.

4 Hsia CC, Herazo LF, Fryder-Doffey F, et al. Compensatory lung growth occurs in adult dogs after right pneumonectomy. J Clin Invest 1994; 94: 405-412.

5 Fehrenbach H, Voswinckel R, Michl V, et al. Neoalveolarisation contributes to compensatory lung growth following pneumonectomy in mice. Eur Respir J 2008; 31: 515-522.

6 Massaro GD, Radaeva S, Clerch LB, et al. Lung alveoli: endogenous programmed destruction and regeneration. Am J Physiol Lung Cell Mol Physiol 2002; 283: L305-L309.

7 Voswinckel R, Motejl V, Fehrenbach A, et al. Characterisation of post-pneumonectomy lung growth in adult mice. Eur Respir J 2004; 24: 524-532.

8 R Development Core Team R. A language and environment for statistical computing. R Foundation for statistical computing, Vienna, Austria, 2007.

9 Smyth G. Limma: linear models for microarray data. In: Gentleman R CV, Dudoit S, Irizarry R, Huber W, eds. Bioinformatics and computational biology solutions using $\mathrm{R}$ and bioconductor. Springer, New York, 2005; pp. 397-420.

10 Gentleman RC, Carey VJ, Bates DM, et al. Bioconductor: open software development for computational biology and bioinformatics. Genome Biol 2004; 5: R80.

11 Edwards D. Non-linear normalization and background correction in one-channel cDNA microarray studies. Bioinformatics 2003; 19: 825-833.

12 Smyth GK, Speed T. Normalization of cDNA microarray data. Methods 2003; 31: 265-273.

13 Smyth GK. Linear models and empirical bayes methods for assessing differential expression in microarray experiments. Stat Appl Genet Mol Biol 2004; 3: article 3.

14 Ferkingstad E, Langaas M, Lindqvist B. Estimating the proportion of true null hypotheses, with application to DNA microarray data. J R Stat Soc Series B Stat Methodol 2005; 67: 555-572.

15 Fink L, Kohlhoff S, Stein MM, et al. cDNA array hybridization after laser-assisted microdissection from nonneoplastic tissue. Am J Pathol 2002; 160: 81-90. 
16 Fink L, Stahl U, Ermert L, et al. Rat porphobilinogen deaminase gene: a pseudogene-free internal standard for laser-assisted cell picking. Biotechniques 1999; 26: 510-516.

17 Livak KJ, Schmittgen TD. Analysis of relative gene expression data using real-time quantitative PCR and the $2(-\Delta \Delta C(T))$ Method. Methods 2001; 25: 402-408.

18 Mariani TJ, Reed JJ, Shapiro SD. Expression profiling of the developing mouse lung: insights into the establishment of the extracellular matrix. Am J Respir Cell Mol Biol 2002; 26: 541-548.

19 Foster JJ, Goss KL, George CL, et al. Galectin-1 in secondary alveolar septae of neonatal mouse lung. Am J Physiol Lung Cell Mol Physiol 2006; 291: L1142-L1149.

20 Boucherat O, Franco-Montoya ML, Thibault C, et al. Gene expression profiling in lung fibroblasts reveals new players in alveolarization. Physiol Genomics 2007; 32: 128-141.

21 Clerch LB, Baras AS, Massaro GD, et al. DNA microarray analysis of neonatal mouse lung connects regulation of KDR with dexamethasone-induced inhibition of alveolar formation. Am J Physiol Lung Cell Mol Physiol 2004; 286: L411-L419.

22 Massaro D, Massaro GD, Baras A, et al. Calorie-related rapid onset of alveolar loss, regeneration, and changes in mouse lung gene expression. Am J Physiol Lung Cell Mol Physiol 2004; 286: L896-L906.

23 Massaro D, Alexander E, Reiland K, et al. Rapid onset of gene expression in lung, supportive of formation of alveolar septa, induced by refeeding mice after calorie restriction. Am J Physiol Lung Cell Mol Physiol 2007; 292: L1313-L1326.

24 Steiglitz BM, Keene DR, Greenspan DS. PCOLCE2 encodes a functional procollagen C-proteinase enhancer (PCPE2) that is a collagen-binding protein differing in distribution of expression and post-translational modification from the previously described PCPE1. J Biol Chem 2002; 277: 49820-49830.

25 Ding X, Kaminsky LS. Human extrahepatic cytochromes P450: function in xenobiotic metabolism and tissue-selective chemical toxicity in the respiratory and gastrointestinal tracts. Annu Rev Pharmacol Toxicol 2003; 43: 149-173.

26 Yildirim AO, Veith $\mathrm{M}$, Rausch $\mathrm{T}$, et al. Keratinocyte growth factor protects against Clara cell injury induced by naphthalene. Eur Respir J 2008; 32: 694-704.

27 Reynolds SD, Zemke AC, Giangreco A, et al. Conditional stabilization of beta-catenin expands the pool of lung stem cells. Stem Cells 2008; 26: 1337-1346.

28 Akerfelt M, Trouillet D, Mezger V, et al. Heat shock factors at a crossroad between stress and development. Ann N Y Acad Sci 2007; 1113: 15-27.
29 Sunil VR, Patel KJ, Nilsen-Hamilton M, et al. Acute endotoxemia is associated with upregulation of lipocalin $24 \mathrm{p} 3 / \mathrm{Lcn} 2$ in lung and liver. Exp Mol Pathol 2007; 83: 177-187.

30 Gwira JA, Wei F, Ishibe S, et al. Expression of neutrophil gelatinase-associated lipocalin regulates epithelial morphogenesis in vitro. J Biol Chem 2005; 280: 7875-7882.

31 Mason N, Ciufo LF, Brown JD. Elongation arrest is a physiologically important function of signal recognition particle. EMBO J 2000; 19: 4164-4174.

32 Hamacher-Brady A, Brady NR, Gottlieb RA. The interplay between pro-death and pro-survival signaling pathways in myocardial ischemia/reperfusion injury: apoptosis meets autophagy. Cardiovasc Drugs Ther 2006; 20: 445-462.

33 Maruyama R, Goto K, Takemura G, et al. Morphological and biochemical characterization of basal and starvation-induced autophagy in isolated adult rat cardiomyocytes. Am J Physiol Heart Circ Physiol 2008; 295: H1599-H1607.

34 Adamson ED, Mercola D. Egr1 transcription factor: multiple roles in prostate tumor cell growth and survival. Tumour Biol 2002; 23: 93-102.

35 O'Donovan KJ, Tourtellotte WG, Millbrandt J, et al. The EGR family of transcription-regulatory factors: progress at the interface of molecular and systems neuroscience. Trends Neurosci 1999; 22: 167-173.

36 Yan SF, Fujita T, Lu J, et al. Egr-1, a master switch coordinating upregulation of divergent gene families underlying ischemic stress. Nat Med 2000; 6: 1355-1361.

37 Sureban SM, Ramalingam S, Natarajan G, et al. Translation regulatory factor RBM3 is a proto-oncogene that prevents mitotic catastrophe. Oncogene 2008; 27: 4544-4556.

38 Adams D, Larman B, Oxburgh L. Developmental expression of mouse follistatin-like 1 (Fstl1): dynamic regulation during organogenesis of the kidney and lung. Gene Expr Patterns 2007; 7: 491-500.

39 Bonapace IM, Latella L, Papait R, et al. Np95 is regulated by E1A during mitotic reactivation of terminally differentiated cells and is essential for S phase entry. J Cell Biol 2002; 157: 909-914.

40 Erdogan M, Pozzi A, Bhowmick N, et al. Signaling pathways regulating TC21-induced tumorigenesis. J Biol Chem 2007; 282: 27713-27720.

41 Sers C, Emmenegger U, Husmann K, et al. Growth-inhibitory activity and downregulation of the class II tumor-suppressor gene H-rev107 in tumor cell lines and experimental tumors. J Cell Biol 1997; 136: 935-944. 